\title{
Choice of Steerable Sheath Impacts Contact Force Stability During Pulmonary Vein Isolation
}

\author{
Evan Hiner ${ }^{1}$ and Dipak Shah ${ }^{2}$ \\ ${ }^{1}$ Advocate Christ Medical Center \\ ${ }^{2}$ Providence Hospital
}

November 19, 2020

\begin{abstract}
Purpose: A stable contact force (CF) is correlated with more effective radiofrequency ablation (RFA) lesions and long-term procedural outcomes. Efforts to improve catheter stability include jet ventilation, pacing, steerable sheaths, and CF sensing ablation catheters. This study compares CF stability and effective RF lesions between two commercially available steerable sheaths. Methods: Thirty patients underwent first time RFA at a single center using the Agilis NxT or SureFlex Steerable Sheath. High power short duration RFA was utilized targeting a $10 \Omega$ drop. Sheath performance was assessed for the entire procedure and around each pulmonary vein (PV) in terms of mean CF, CF variability, RF time per lesion, and inefficient contact lesions (defined as lesions with $\mathrm{CF}<5 \mathrm{~g}$ for at least $10 \%$ of the RF delivery time). Results: Operator-targeted mean $\mathrm{CF}$ was achieved using both sheaths; however, overall CF variability was $12.8 \%$ lower using the SureFlex sheath $(\mathrm{p}=0.08)$. CF variability was generally $16 \%$ greater in the right PVs than the left PVs $(p=0.001)$, but trended lower with the SureFlex sheath. There were $8 \%$ more inefficient contact lesions using Agilis as compared to SureFlex $(\mathrm{p}=0.035)$, especially in the right inferior PV $(\mathrm{p}=0.009)$. RF time per lesion was on average $12 \%(1.4 \mathrm{~s})$ shorter using SureFlex than Agilis $(\mathrm{p}<0.05)$. Conclusion: Choice of steerable sheath may affect catheter stability and potential lesion quality, especially in the right PVs.
\end{abstract}

\section{Title:}

Choice of Steerable Sheath Impacts Contact Force Stability During Pulmonary Vein Isolation Authors: Evan Hiner, $\mathrm{MD}^{1,2}$ and Dipak P. Shah, $\mathrm{MD}^{2}$

\section{Affiliations:}

1) Michigan State University, College of Human Medicine

2) Ascension Health, Providence Hospital and Medical Center

Corresponding author: Dipak P. Shah

22250 Providence Dr \#705 Southfield, MI 48075 dipak.shah@ascension.org

\section{Acknowledgement/ Financial support:}

None.

Disclosures:

Dr. Shah is on the Speaker's Bureau for Janssen Pharmaceuticals and Bristol-Myers Squibb.

\section{Abstract}


Purpose: A stable contact force (CF) is correlated with more effective radiofrequency ablation (RFA) lesions and long-term procedural outcomes. Efforts to improve catheter stability include jet ventilation, pacing, steerable sheaths, and CF sensing ablation catheters. This study compares CF stability and effective RF lesions between two commercially available steerable sheaths.

Methods: Thirty patients underwent first time RFA at a single center using the Agilis NxT or SureFlex Steerable Sheath. High power short duration RFA was utilized targeting a $10 \Omega$ drop. Sheath performance was assessed for the entire procedure and around each pulmonary vein (PV) in terms of mean $\mathrm{CF}$, CF variability, $\mathrm{RF}$ time per lesion, and inefficient contact lesions (defined as lesions with $\mathrm{CF}<5 \mathrm{~g}$ for at least $10 \%$ of the RF delivery time).

Results: Operator-targeted mean $\mathrm{CF}$ was achieved using both sheaths; however, overall CF variability was $12.8 \%$ lower using the SureFlex sheath $(\mathrm{p}=0.08)$. CF variability was generally $16 \%$ greater in the right PVs than the left PVs $(\mathrm{p}=0.001)$, but trended lower with the SureFlex sheath. There were $8 \%$ more inefficient contact lesions using Agilis as compared to SureFlex $(\mathrm{p}=0.035)$, especially in the right inferior PV $(\mathrm{p}=$ 0.009). RF time per lesion was on average 12\% (1.4s) shorter using SureFlex than Agilis ( $<<0.05)$.

Conclusion: Choice of steerable sheath may affect catheter stability and potential lesion quality, especially in the right PVs.

Keywords: pulmonary vein isolation, ablation, atrial fibrillation, contact force, catheter stability, steerable sheath

\section{Introduction}

While radiofrequency (RF) catheter ablation for atrial fibrillation (AF) is associated with acute pulmonary vein isolation (PVI) in over $90 \%$ of cases, long-term success of a single ablation for paroxysmal AF has been reported to be $69 \%$ at one year ${ }^{1}$ and only $54 \%$ beyond three years ${ }^{2}$. Long-term PVI success, defined by the reduced rate of AF recurrence, is largely driven by the durability, as well as continuity and transmurality of formed lesions ${ }^{3}$. Acute lesion assessment is based on impedance drop from baseline, change in electrogram amplitude or morphology, change in pacing threshold and/or real-time MRI, while overall procedural success is confirmed in terms of PVI. Durability of RF lesions has been associated with the amount of RF energy delivered. Insufficient RF delivery leads to PV reconnection and prolonged procedure times, while excessive RF application may lead to complications such as esophageal injury, atrial perforation, steam pops and coagulum formation ${ }^{4-6}$. The relative RF energy received by the tissue and, consequently, lesion quality, are dependent on consistent coupling between the ablation catheter tip and the target tissue ${ }^{4,7}$, which can be compromised by small inadvertent movements during the procedure ${ }^{8}$. Efforts to improve catheter-tissue contact and stability include the use of high-frequency jet ventilation to minimize respiratory excursion, high rate pacing to regulate cardiac contractions, electroanatomic mapping to monitor catheter proximity and tissue contact, as well as sheath and catheter selection ${ }^{9-14}$. Introduction of contact force (CF)-sensing ablation catheters has allowed real-time measurement of catheter-tissue contact to guide RF delivery and formation of more effective lesions ${ }^{7}$. The use of $\mathrm{CF}$-sensing catheters has reduced procedure time and fluoroscopy usage, and improved the 1-year AF recurrence rate by up to $12 \%$ compared to non-CF sensing catheters 15-17. The adoption of steerable sheaths in PVI procedures has not only improved catheter manipulation and access to target sites ${ }^{14,18,19}$ but, also, improved procedural CF. In randomized studies, steerable sheaths significantly enhanced CF stability, facilitated mapping and ablation, reduced procedure times and improved procedural efficiency when compared to standard fixed curve sheaths ${ }^{20}$. Improvements in CF using steerable sheaths were further noted in different locations in the left atrium (LA). While accessing the left superior and inferior pulmonary veins requires a relatively straight sheath trajectory, the right superior and inferior pulmonary veins require greater sheath manipulation with tight angles of curvature and stabilization to maintain catheter tip position at the desired location ${ }^{14}$. Steerable sheaths have also been shown to have fewer lesions with insufficient tissue $\mathrm{CF}^{15,21}$; the reduction of these poor $\mathrm{CF}$ lesions has been associated with improved freedom from recurrence at one year ${ }^{21}$. In an effort to optimize catheter stability during PVI 
procedures, the present study compares whether $\mathrm{CF}$ and procedural efficiency can be further improved using different commercially-available steerable sheaths.

\section{Materials and Methods}

\section{Study design}

A retrospective analysis was performed on thirty consecutive RFA procedures conducted at a single center by a single operator (DPS) between February and June 2019. Patients with history of previous ablation or cardiac surgery, as well as cardiac implants, were excluded from the series. Fifteen consecutive patients undergoing PVI procedures using the Agilis NxT Steerable Introducer (Abbott, WS, USA) were compared to 15 consecutive patients using the SureFlex Steerable Guiding Sheath (Baylis Medical, Montreal, CA). Standard informed consent was obtained prior to each procedure. Institutional approval was obtained for retrospective chart review.

\section{RF Ablation Procedure}

Procedures were performed under general anesthesia and as per usual protocol. Percutaneous access was obtained from the right femoral vein for all catheters. Intravenous heparin was administered to maintain an activated clotting time of approx. 350s. Transseptal puncture was performed under ICE and fluoroscopy guidance using the NRG Transseptal Needle (Baylis Medical, Montreal, Canada) with the TorFlex Transseptal Guiding Sheath (Baylis Medical) or SL-1 Transseptal Guiding Introducer (Abbott, ). 3D electroanatomic mapping (EnSite Precision Mapping System, Abbott) was used for catheter guidance and CF measurement. Attempts to stabilize the ablation catheter were limited to the choice of steerable sheath. The Agilis NxT (Abbott, Chicago, IL) or SureFlex steerable sheath (Baylis Medical) were used to position the ablation catheter (TactiCath Contact Force Ablation Catheter, Abbott) for point-by-point ablation using approx. $50 \mathrm{~W}$ RF energy and a target contact force of 10-15g until an impedance drop of approx. 10 was achieved. The left superior and inferior PVs (LSPV and LIPV, respectively) were isolated before attempting to isolate the right superior and inferior PVs (RSPV and RIPV, respectively). Esophageal temperature was monitored during the ablation to remain below approx. $38^{\circ} \mathrm{C}$. Acute PV reconnection was assessed by monitoring electrocardiograms (ECG) and voltage maps for re-appearance of PV potentials within an approx. 10-minute wait time. ECG was performed during follow up of at least 6 months to assess recurrence of symptoms and long-term procedural outcomes.

\section{Data analysis}

CF was recorded by the EnSite mapping system at approx. 10ms intervals during each episode of RF application and were exported from the mapping system for further analysis. RF time per lesion and mean CF per lesion were used to assess procedural efficiency. Stability in CF was evaluated in terms of $\mathrm{CF}$ variability within an individual lesion; standard deviation was calculated for the mean CF within each lesion (Figure 1). Inefficient contact lesions were defined as those whereby CF dropped below the minimum threshold of $5 \mathrm{~g}$ for at least $10 \%$ of the total RF application time for the lesion ${ }^{15,21}$. Analyses were performed for the overall procedure, as well as by correlating each lesion to individual PVs on the electroanatomic map.

\section{Statistical Analysis}

Baseline patient characteristics were compared using a T-test between the two steerable sheath groups. A hierarchical regression analysis was performed for the procedural parameters (mean CF, RF time per lesion, $\mathrm{CF}$ variability) using a linear-mixed effect model by adjusting for individual lesions and each patient on $\mathrm{R}$ software (version 1.1, RStudio Inc, Boston, MA). Time-sequence data to determine inefficient contact lesions was analysed using MATLAB (version 9.4, MathWorks, Natick, MA); significance was evaluated using a F-test on R.

\section{Results}

Contact force analysis by location 
A total of 3157 lesions were analyzed with a mean of $105.2 \pm 33.1$ lesions per patient. Overall, mean CF achieved during ablation of right-sided pulmonary veins was $17 \%$ higher $(\mathrm{p}=0.001$; Figure $2 \mathrm{~A})$ than for leftsided pulmonary veins. CF variability within each lesion was $19 \%$ higher in right-sided veins than left-sided veins $(\mathrm{p}=0.001$; Figure $2 \mathrm{~B})$.

\section{Contact force analysis by steerable sheath}

Baseline patient characteristics were similar in both sheath groups except for significantly higher BMI (p $=0.01)$ and females $(\mathrm{p}=0.03)$ in the SureFlex group than Agilis (Table 1). A total of 1354 lesions were analyzed in the Agilis group and 1803 lesions in the SureFlex group. There was no significant difference in the mean CF between the Agilis sheath and SureFlex sheath at the overall procedure level, as well as around each PV (Table 2). However, CF variability was 13\% lower with the SureFlex sheath over the entire procedure compared to Agilis sheath $(\mathrm{p}=0.043$; Figure 3A). Further analysis showed a trend of $13-14 \%$ lower $\mathrm{CF}$ variability in the left and right superior pulmonary veins with the SureFlex sheath than Agilis sheath (significant at the $\alpha=0.1$ level; Figure 3B).

\section{Inefficient contact lesions}

The SureFlex sheath maintained better catheter-tissue contact than the Agilis sheath, as demonstrated by a $20 \%$ overall fewer inefficient contact lesions (i.e. lesions with $\mathrm{CF}<5 \mathrm{~g}$ for more than $10 \%$ of the ablation time; $\mathrm{p}<0.001$, Figure 4A). The odds ratio (OR) for inefficient contact lesions was 0.605 for SureFlex versus Agilis (95\% CI: 0.371-0.976, $\mathrm{p}=0.035)$, revealing a $39 \%$ lower chance of inefficient contact lesions with the SureFlex sheath than Agilis. While this reduction was consistent among all pulmonary veins, the difference between SureFlex and Agilis was significant in the right PVs, with $17 \%$ and $45 \%$ fewer inefficient contact lesions in the right inferior and right superior PVs, respectively $(\mathrm{p}<0.01$; Figure $4 \mathrm{~B})$. OR for SureFlex sheath versus Agilis for RIPV was 0.607 (95\% CI: 0.35-1.03; $\mathrm{p}=0.009$ ) and RSPV was 0.583 (95\% CI: $0.27-1.24 ; \mathrm{p}=0.15)$.

\section{RF time per lesion}

Overall RF ablation time per lesion was $12 \%$ (1.4s per lesion) shorter with SureFlex sheath when compared to Agilis sheath $(\mathrm{p}=0.002$; Figure $5 \mathrm{~A})$. A $9-21 \%$ reduction in $\mathrm{RF}$ ablation time was observed in the left superior, right inferior and right superior pulmonary veins when using the SureFlex sheath compared to Agilis $(\mathrm{p}<0.05$; Figure 5B).

\section{Procedural outcomes}

PVI was successfully achieved in all 30 patients with paroxysmal (57\%) and persistent (37\%) AF. No major procedure-related adverse events, such as cardiac tamponade, stroke or esophageal injury, occurred. Patients seen in follow-up up to 6 months post-ablation remained in sinus rhythm. Anti-arrhythmic drug use at 6 months post-ablation was continued in $53 \%$ of patients in the Agilis group and only $27 \%$ of patients in the SureFlex group; however, this did not reach statistical significance.

\section{Discussion}

Permanent PVI and freedom from AF recurrence are contingent upon efficient and durable RF lesion formation ${ }^{22,23}$. The amount of RF energy delivered to the tissue during ablation plays a central role in lesion durability ${ }^{24}$. Good energy coupling from the ablation catheter to the cardiac tissue requires stable catheter-tissue contact with sufficient $\mathrm{CF}$ during RF application ${ }^{25}$. Catheter stability, adequate $\mathrm{CF}$ and maximum time spent in the desired contact force range have been correlated with reduced PV reconnection and improved RF ablation outcomes ${ }^{11,21,26}$. Use of steerable sheaths has improved procedural outcomes in comparison to conventional sheaths due to the ease of manipulation, access and contact with target sites ${ }^{14,19}$. The rigid make up of steerable sheaths, also, reduces the effects of cardiac and respiratory excursions and provide better catheter control ${ }^{23}$. Several steerable sheaths are commercially available but their relative influence on $\mathrm{CF}$ and catheter stability remains unknown. In this study, retrospective evaluation of data from 30 cases performed using two different steerable sheaths indicated that procedural efficiency measures such 
as CF stability and RF time per lesion may be impacted by the choice of sheath. A consistent ablation strategy was used whereby pulmonary veins were ablated in sequence, with the left veins before the right veins, suggesting that any observed trends are attributed to both anatomy and/or sheath fatigue over time.

Both steerable sheaths in this study achieved similar mean CF, ruling out potential procedural bias. However, it has become evident that catheter and CF stability may play more important roles in forming effective lesions with lower reconnection rates than $\mathrm{CF}$ magnitude alone ${ }^{4}$. In this case series, the SureFlex sheath maintained an overall $13 \%$ greater CF stability than the Agilis sheath, with similar trends in each pulmonary vein.

In studies targeting a CF of approx. $20 \mathrm{~g}$, it was found that maintaining a minimum $\mathrm{CF}$ of $10 \mathrm{~g}$ for at least $80 \%$ of the individual RF application time was necessary for effective lesions and significantly improved overall ablation outcomes ${ }^{15,21}$. In contrast, RF application with insufficient tissue $\mathrm{CF}$ has been correlated with inadequate lesion formation ${ }^{26-28}$ and higher rate of $\mathrm{AF}$ recurrence ${ }^{21,22,29}$. Since a higher power ablation protocol was used in the present study, a lower target CF (10-15g) and minimum threshold for catheter tissue contact $(5 \mathrm{~g})$ were defined ${ }^{27}$. The SureFlex sheath led to significantly fewer lesions with poor contact $(<5 \mathrm{~g}$ for more than $80 \%$ of the ablation time). This trend was further pronounced in the right inferior and superior PVs, which are typically more difficult to navigate.

Reduced CF stability may necessitate longer RF application time in order to reach the desired lesion endpoint and acute success ${ }^{4,24}$. This not only reduces overall efficiency and prolongs procedure time but, also, increases the risk of coagulum formation and steam pops due to excessive RF application ${ }^{28,30}$. In this study, RF time per lesion was $12 \%$ shorter in the SureFlex group than Agilis, suggesting improved RF delivery and procedural efficiency. To our knowledge, this is the first study that compares the procedural performance of two commercially available sheaths with the goal of improving CF stability for more effective RF ablations. Preliminary data from follow-up visits suggests fewer patients in the SureFlex group remained on antiarrhythmic drugs at 6 months post-ablation than in the Agilis group; however, this did not reach significance.

\section{Limitations:}

This single-operator non-randomized study retrospectively evaluated procedural parameters from a small patient population, which limited the statistical power of the analyses. While a consecutive series of patients undergoing first-time ablation was selected, BMI and female population were higher in the SureFlex group, which may have introduced procedural complexity and technical challenges. The operator was not blinded to the sheath being used; however, similar mean CF achieved using both sheaths is indicative of a lack of operator bias. Furthermore, limited follow-up information was available due to the small patient cohort. Larger randomized studies involving multiple operators and longer patient follow-up are needed to validate these findings and understand the effects of CF stability on procedural outcomes. Other commercially available sheaths, as well as the contribution of different ablation catheters, can also be explored to optimize CF stability.

\section{Conclusions}

CF variability can produce suboptimal lesions that lead to pulmonary vein reconnection. This study demonstrates that the choice of steerable sheath may improve CF stability and consistency over time.

\section{References}

1. Murray MI, Arnold A, Younis M, Varghese S, Zeiher AM. Cryoballoon versus radiofrequency ablation for paroxysmal atrial fibrillation: a meta-analysis of randomized controlled trials. Clin Res Cardiol.2018;107(8):658-669.

2. Ganesan AN, Shipp NJ, Brooks AG, et al. Long-term outcomes of catheter ablation of atrial fibrillation: a systematic review and meta-analysis. J Am Heart Assoc. 2013;2(2):e004549.

3. Hocini M, Condie C, Stewart MT, Kirchhof N, Foell JD. Predictability of lesion durability for AF ablation using phased radiofrequency: Power, temperature, and duration impact creation of transmural lesions. Heart 
Rhythm. 2016;13(7):1521-1526.

4. El Haddad M, Taghji P, Phlips T, et al. Determinants of Acute and Late Pulmonary Vein Reconnection in Contact Force-Guided Pulmonary Vein Isolation: Identifying the Weakest Link in the Ablation Chain.Circulation: Arrhythmia and Electrophysiology.2017;10(4):e004867.

5. Kottmaier M, Popa M, Bourier F, et al. Safety and outcome of very high-power short-duration ablation using $70 \mathrm{~W}$ for pulmonary vein isolation in patients with paroxysmal atrial fibrillation.Europace. 2020;22(3):388-393.

6. Reddy VY, Pollak S, Lindsay BD, et al. Relationship Between Catheter Stability and 12-Month Success After Pulmonary Vein Isolation: A Subanalysis of the SMART-AF Trial. JACC Clin Electrophysiol.2016;2(6):691-699.

7. Kumar S, Barbhaiya CR, Balindger S, et al. Better Lesion Creation And Assessment During Catheter Ablation. J Atr Fibrillation.2015;8(3):1189.

8. Rafael A, Heist EK. Techniques to Optimize Catheter Contact Force during Ablation of Atrial Fibrillation. J Innovations in Cardiac Rhythm Management. 2015;6(May):1990-1995.

9. Okumura Y, Watanabe I, Kofune M, et al. Effect of catheter tip-tissue surface contact on threedimensional left atrial and pulmonary vein geometries: potential anatomic distortion of 3D ultrasound, fast anatomical mapping, and merged 3D CT-derived images. J Cardiovasc Electrophysiol. 2013;24(3):259-266.

10. Goode JS, Jr., Taylor RL, Buffington CW, Klain MM, Schwartzman D. High-frequency jet ventilation: utility in posterior left atrial catheter ablation. Heart Rhythm. 2006;3(1):13-19.

11. Hutchinson MD, Garcia FC, Mandel JE, et al. Efforts to enhance catheter stability improve atrial fibrillation ablation outcome.Heart Rhythm. 2013;10(3):347-353.

12. Aizer A, Cheng AV, Wu PB, et al. Pacing Mediated Heart Rate Acceleration Improves Catheter Stability and Enhances Markers for Lesion Delivery in Human Atria During Atrial Fibrillation Ablation. JACC Clin Electrophysiol. 2018;4(4):483-490.

13. Ali A, Plettenburg DH, Breedveld P. Steerable Catheters in Cardiology: Classifying Steerability and Assessing Future Challenges.IEEE Trans Biomed Eng. 2016;63(4):679-693.

14. Piorkowski C, Eitel C, Rolf S, et al. Steerable versus nonsteerable sheath technology in atrial fibrillation ablation: a prospective, randomized study. Circ Arrhythm Electrophysiol.2011;4(2):157-165.

15. Deyell MW, Wen G, Laksman Z, et al. The impact of steerable sheaths on unblinded contact force during catheter ablation for atrial fibrillation. J Interv Card Electrophysiol. 2019.

16. Marijon E, Fazaa S, Narayanan K, et al. Real-time contact force sensing for pulmonary vein isolation in the setting of paroxysmal atrial fibrillation: procedural and 1-year results. J Cardiovasc Electrophysiol. 2014;25(2):130-137.

17. Andrade JG, Monir G, Pollak SJ, et al. Pulmonary vein isolation using "contact force" ablation: the effect on dormant conduction and long-term freedom from recurrent atrial fibrillation - a prospective study. Heart Rhythm. 2014;11(11):1919-1924.

18. Masuda M, Fujita M, Iida O, et al. Steerable versus non-steerable sheaths during pulmonary vein isolation: impact of left atrial enlargement on the catheter-tissue contact force. J Interv Card Electrophysiol. 2016;47(1):99-107.

19. Rajappan K, Baker V, Richmond L, et al. A randomized trial to compare atrial fibrillation ablation using a steerable vs. a non-steerable sheath. Europace. 2009;11(5):571-575.

20. Ullah W, Hunter RJ, McLean A, et al. Impact of steerable sheaths on contact forces and reconnection sites in ablation for persistent atrial fibrillation. J Cardiovasc Electrophysiol. 2015;26(3):266-273. 
21. Natale A, Reddy VY, Monir G, et al. Paroxysmal AF catheter ablation with a contact force sensing catheter: results of the prospective, multicenter SMART-AF trial. J Am Coll Cardiol.2014;64(7):647-656.

22. Neuzil P, Reddy VY, Kautzner J, et al. Electrical reconnection after pulmonary vein isolation is contingent on contact force during initial treatment: results from the EFFICAS I study. Circulation: Arrhythmia and Electrophysiology. 2013;6(2):327-333.

23. Pedrote A, Acosta J, Jauregui-Garrido B, Frutos-Lopez M, Arana-Rueda E. Paroxysmal atrial fibrillation ablation: Achieving permanent pulmonary vein isolation by point-by-point radiofrequency lesions. World $J$ Cardiol. 2017;9(3):230-240.

24. Chinitz JS, Kapur S, Barbhaiya C, et al. Sites with small impedance decrease during catheter ablation for atrial fibrillation are associated with recovery of pulmonary vein conduction. Journal of cardiovascular electrophysiology. 2016;27(12):1390-1398.

25. Haines DE. Cooking With Radiofrequency Energy: What Is the Right Recipe? JACC Clin Electrophysiol. 2018;4(4):480-482.

26. Calkins H, Kuck KH, Cappato R, et al. 2012 HRS/EHRA/ECAS expert consensus statement on catheter and surgical ablation of atrial fibrillation: recommendations for patient selection, procedural techniques, patient management and follow-up, definitions, endpoints, and research trial design: a report of the Heart Rhythm Society (HRS) Task Force on Catheter and Surgical Ablation of Atrial Fibrillation. Developed in partnership with the European Heart Rhythm Association (EHRA), a registered branch of the European Society of Cardiology (ESC) and the European Cardiac Arrhythmia Society (ECAS); and in collaboration with the American College of Cardiology (ACC), American Heart Association (AHA), the Asia Pacific Heart Rhythm Society (APHRS), and the Society of Thoracic Surgeons (STS). Endorsed by the governing bodies of the American College of Cardiology Foundation, the American Heart Association, the European Cardiac Arrhythmia Society, the European Heart Rhythm Association, the Society of Thoracic Surgeons, the Asia Pacific Heart Rhythm Society, and the Heart Rhythm Society. Heart Rhythm.2012;9(4):632-696 e621.

27. Ullah W, McLean A, Tayebjee MH, et al. Randomized trial comparing pulmonary vein isolation using the SmartTouch catheter with or without real-time contact force data. Heart Rhythm. 2016;13(9):1761-1767.

28. Vassallo F, Cunha C, Serpa E, et al. Comparison of high-power short-duration (HPSD) ablation of atrial fibrillation using a contact force-sensing catheter and conventional technique: Initial results.J Cardiovasc Electrophysiol. 2019;30(10):1877-1883.

29. Chinitz LA, Melby DP, Marchlinski FE, et al. Safety and efficiency of porous-tip contact-force catheter for drug-refractory symptomatic paroxysmal atrial fibrillation ablation: results from the SMART SF trial. Europace. 2018;20(FI_3):f392-f400.

30. Nakagawa H, Kautzner J, Natale A, et al. Locations of high contact force during left atrial mapping in atrial fibrillation patients: electrogram amplitude and impedance are poor predictors of electrode-tissue contact force for ablation of atrial fibrillation. Circ Arrhythm Electrophysiol. 2013;6(4):746-753.

\section{Tables}

Table 1: Baseline patient demographics

\begin{tabular}{llll}
\hline Characteristic & SureFlex $(\mathrm{n}=15)$ & Agilis $(\mathrm{n}=15)$ & p-value \\
\hline Age (avg, years) & $60.2 \pm 11.9$ & $64.7 \pm 11.0$ & 0.30 \\
Gender (male sex) $(\%)$ & 40 & 80 & 0.025 \\
BMI (avg) & $35.9 \pm 8.8$ & $28.8 \pm 3.6$ & 0.01 \\
Hypertension (\%) & 80 & 67 & 0.43 \\
Diabetes (\%) & 0 & 13 & 0.16 \\
ASCVD (\%) & 40 & 67 & 0.15
\end{tabular}




\begin{tabular}{|c|c|c|c|}
\hline Characteristic & SureFlex $(\mathrm{n}=15)$ & Agilis $(\mathrm{n}=15)$ & p-value \\
\hline Heart failure (\%) & 20 & 40 & 0.25 \\
\hline Pacemaker $(\%)$ & 13 & 0 & 0.16 \\
\hline $\begin{array}{l}\text { Paroxysmal/Persistent } \\
\text { AF }(\%)\end{array}$ & $60 / 27$ & $53 / 47$ & $0.72 / 0.27$ \\
\hline $\begin{array}{l}\text { Ejection fraction } \\
{[?] 50 \%(\%)}\end{array}$ & 21 & 25 & 0.84 \\
\hline LVH $(\%)$ & 33 & 47 & 0.47 \\
\hline $\begin{array}{l}\text { LA size - Volume/BSA } \\
\left(\operatorname{avg}, \mathrm{cm}^{2}\right)\end{array}$ & $38.7 \pm 18.7$ & $39.8 \pm 17.9$ & 0.89 \\
\hline LA dilation $(\%)$ & 53 & 60 & 0.72 \\
\hline $\begin{array}{l}\text { Mitral Regurgitation } \\
(\%)\end{array}$ & 73 & 67 & 0.70 \\
\hline $\begin{array}{l}\text { Oral anti-coagulant use } \\
(\%)\end{array}$ & 100 & 100 & - \\
\hline $\begin{array}{l}\text { Anti-arrhythmic drug } \\
\text { use }(\%)\end{array}$ & 53 & 60 & 0.72 \\
\hline
\end{tabular}

Table 2: Mean contact force per lesion achieved using the Agilis or SureFlex sheath

\begin{tabular}{llll}
\hline & Agilis & SureFlex & p-value \\
\hline Overall & $13.7 \pm 1.3$ & $14.6 \pm 1.8$ & 0.32 \\
LSPV & $13.05 \pm 2.9$ & $13.41 \pm 4.1$ & 0.53 \\
LIPV & $14.04 \pm 3.2$ & $14.38 \pm 4.4$ & 0.57 \\
RSPV & $13.09 \pm 3.5$ & $14.07 \pm 4.9$ & 0.43 \\
RIPV & $14.73 \pm 3.3$ & $16.78 \pm 4.5$ & 0.43 \\
\hline
\end{tabular}

\section{Figures}

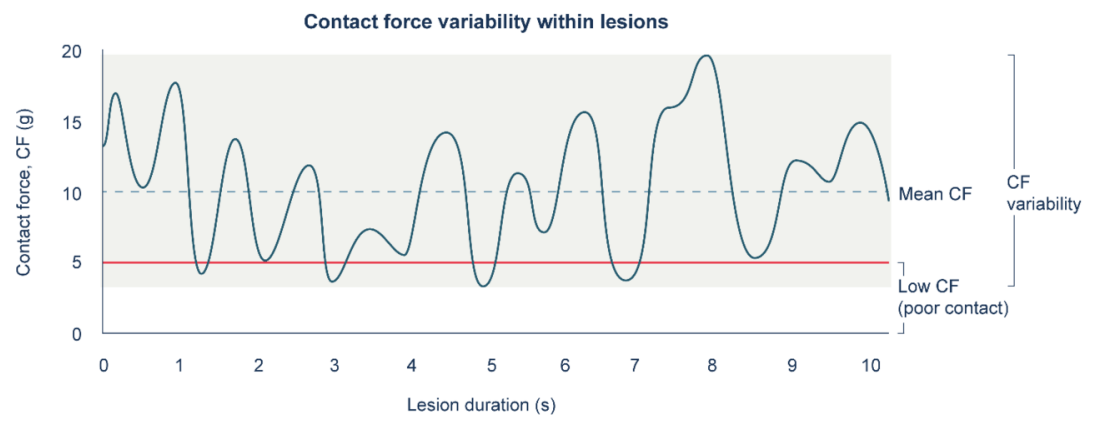

Figure 1. Analysis of contact force (CF) parameters. Small inadvertent movements contribute to catheter instability and fluctuations in contact force $(\mathrm{CF})$. Measurements obtained from the EAM system were used to assess mean $\mathrm{CF}$ and $\mathrm{CF}$ variability for each lesion. Poor contact was considered when $\mathrm{CF}$ 
dropped below $5 \mathrm{~g}$; lesions with poor contact for more than $10 \%$ of the RF application time were defined as "inefficient contact lesions."
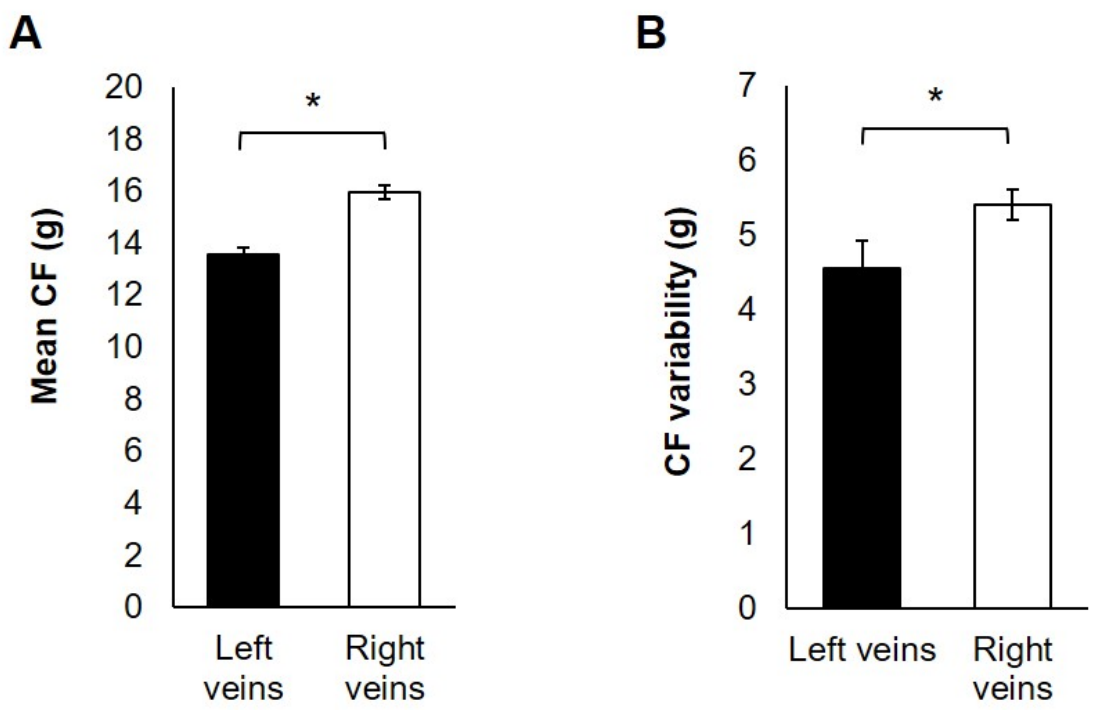

Figure 2. Comparison of contact force in left vs. right pulmonary veins. A) Higher mean contact force per lesion was achieved among right-sided pulmonary veins (i.e. right inferior and superior) than leftsided veins (i.e. left inferior and superior). B) A higher level of $\mathrm{CF}$ variability was observed among individual lesions in right-sided veins than left sided veins. $\left({ }^{*} \mathrm{p}=0.001\right)$.

A

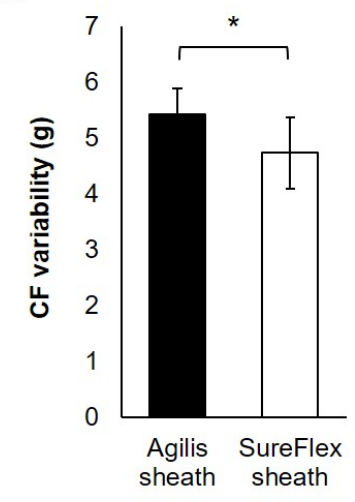

B

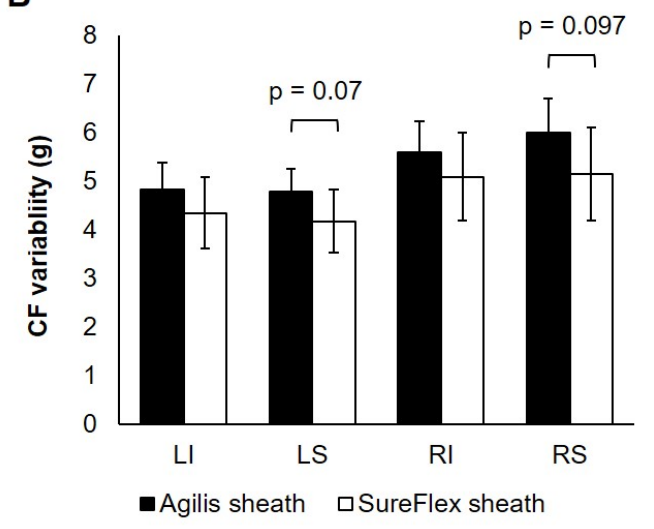

Figure 3. Analysis of CF variability within individual ablation lesions. A) Significantly lower CF variability was found using the SureFlex sheath than Agilis. B) Similar trends were observed in each of the pulmonary veins with lower CF variability using the SureFlex sheath; however, this did not reach statistical significance. $\left({ }^{*} \mathrm{p}=0.043\right)$. 
A

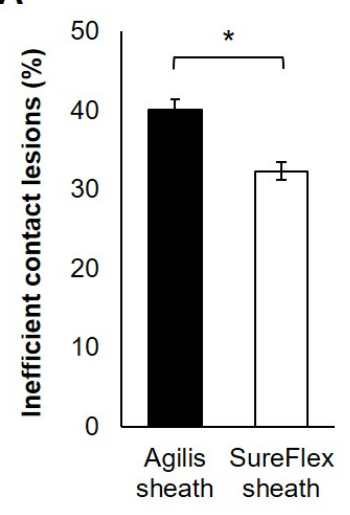

B

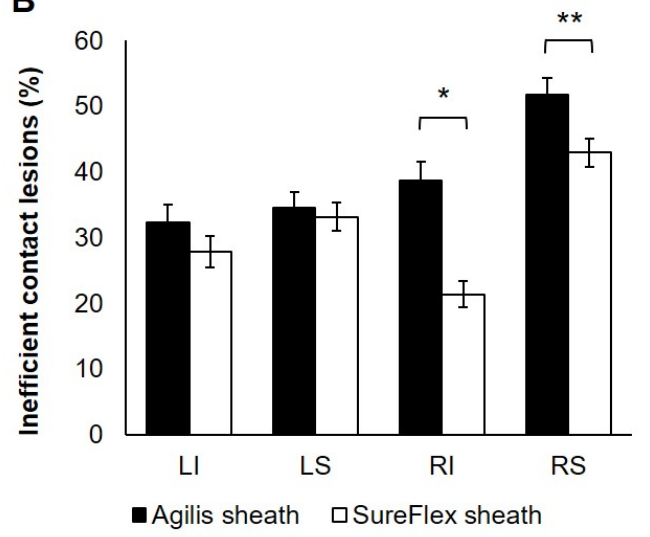

Figure 4. Inefficient contact lesions with greater than $10 \%$ of the ablation time below the minimum acceptable $\mathbf{C F}(\mathbf{C F}<\mathbf{5 g})$. A) Fewer inefficient contact lesions were found in the SureFlex group than Agilis. B) Difficult-to-access right-sided (i.e. RI and RS) pulmonary veins had the greatest difference in inefficient contact lesions between the SureFlex and Agilis groups. $\left({ }^{*} \mathrm{p}<0.001,{ }^{* *} \mathrm{p}=0.009\right)$.
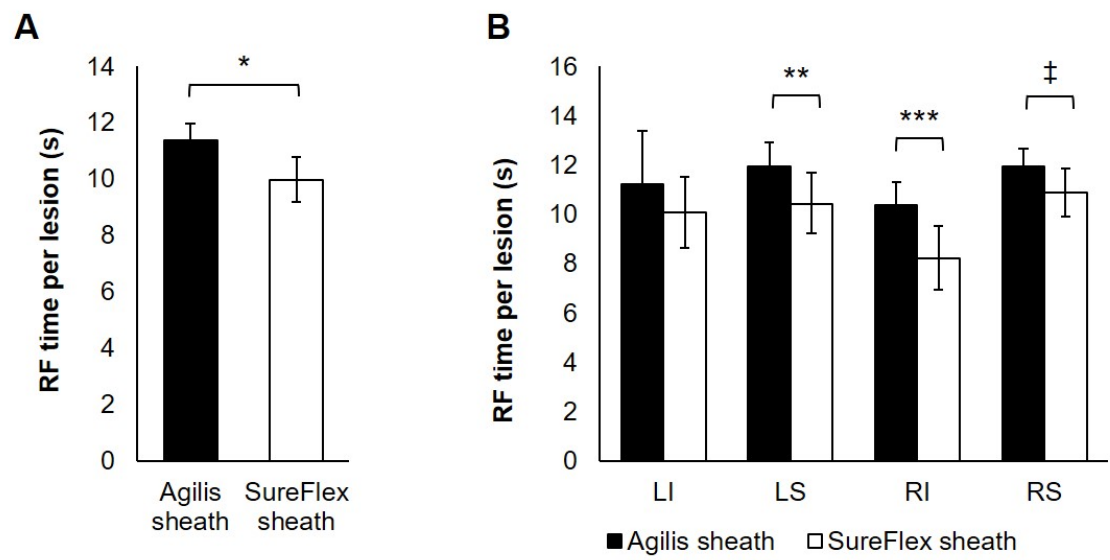

Figure 5. RF time per lesion. A) RF ablation time to reach the acute lesion endpoint (i.e. impedance drop of approx. 10) was $12 \%$ shorter in the SureFlex group than the Agilis group. B) RF time per lesion was $9-21 \%$ lower in the LS, RI and RS pulmonary veins when using the SureFlex sheath. $\left({ }^{*} \mathrm{p}=0.002,{ }^{* *} \mathrm{p}\right.$ $\left.=0.022,{ }^{* * *} \mathrm{p}=0.003,{ }^{++} \mathrm{p}=0.048\right)$. 